\title{
Connaissances Et Motivations Des Medecins Dans La Prescription Des Examens D'imagerie Devant Une Urgence Abdominale Non Traumatique Au Togo: A Propos D'une Enquete Realisee Aupres Des Medecins
}

\author{
Amadou A. \\ Agbangba KA. \\ Watara $G$. \\ Sonhaye L.
}

Service de Radiologie et Imagerie du CHU Campus.

Tchangaï $B$.

Service de Chirurgie Générale du CHU Sylvanus Olympio.

Tchaou M.

Service de Radiologie et Imagerie du CHU Sylvanus Olympio.

\section{Adjenou $V$. \\ N'dakena $K$.}

Service de Radiologie et Imagerie du CHU Campus.

\begin{abstract}
Objective: To evaluate physicians' knowledge of prescribing imaging modalities in non-traumatic abdominal emergencies. Materials and method: A transversal and analytical study, consisting of the filling of a questionnaire distributed to general physicians. The survey card included, among others, the first-line imaging modality and the most effective on four abdominal emergencies found in Togo. Results: Regarding acute appendicitis, 73\% of physicians estimated the imaging needed. For $92 \%$, ultrasound was the firstline imaging technique because of its availability according to $40 \%$. For $87 \%$, the scan was the most effective exam. In peritonitis, $69 \%$ of physicians considered imaging important. For $98 \%$ Conventional Chest and Abdominal Radiography was the first-line examination because $52 \%$ of physicians said it was the most available. $89 \%$ thought the CT scan was the most effective. In the case of bowel obstruction, $89 \%$ of physicians found the necessary imaging. For $96 \%$, the first-line examination was the Conventional Chest and Abdominal Radiography because of its availability according to $51 \%$ of doctors. All physicians felt that the CT scan was the most effective
\end{abstract}


examination. Concerning acute cholecystitis, 95\% considered imaging very important. For 95\%, ultrasound was the first-line examination. Its prescription was motivated by its availability according to $50 \%$ of doctors. $96 \%$ felt that CT was the most effective examination. Conclusion: From our study, it appears that in abdominal emergencies, imaging examinations necessary for diagnosis are prescribed according to their availability and cost, not according to their effectiveness.

Keywords: Non-traumatic abdominal emergency - First-line imaging - Most effective imaging - Choice of imaging - Rationale for choosing imaging.

\section{Resume}

Objectifs : Evaluer la connaissance des médecins sur la prescription des examens d'imagerie dans les urgences abdominales non traumatiques. Matériels et méthode : Etude transversale et analytique, ayant consisté au remplissage d'un questionnaire distribué aux médecins généralistes et aux internes. La fiche d'enquête portait entre autres sur les examens d'imagerie de première intention et le plus efficace sur quatre pathologies d'urgence abdominale retrouvées au Togo. Résultats : Concernant l'appendicite aiguë, $73 \%$ des médecins estimaient l'imagerie nécessaire. Pour 92\%, l'échographie était l'examen de première intention du fait de sa disponibilité selon $40 \%$. Pour $87 \%$, le scanner était l'examen le plus efficace. Dans les péritonites, $69 \%$ des médecins considéraient l'imagerie importante. Pour 98\% l'ASP était l'examen de première intention, car selon $52 \%$ des médecins il est le plus disponible. $89 \%$ estimaient que le scanner était l'examen le plus efficace. En cas d'occlusions intestinales, $89 \%$ des médecins trouvaient l'imagerie nécessaire. Pour 96\%, l'examen de première intention était l'ASP du fait de sa disponibilité selon $51 \%$ des médecins Tous les médecins estimaient que le scanner était l'examen le plus efficace. Concernant les cholécystites aiguës, $95 \%$ considéraient l'imagerie très importante. Pour $95 \%$, l'échographie était l'examen de première intention. Sa prescription était motivée par sa disponibilité selon $50 \%$ des médecins. 96\% estimaient que le scanner était l'examen le plus efficace. Conclusion : De notre étude, il ressort que dans les urgences abdominales, les examens d'imagerie nécessaires au diagnostic, sont prescrits selon leur disponibilité et leur coût, et non selon leur efficacité.

Mots clés : Urgence abdominale non traumatique - Imagerie de première intention - Imagerie le plus efficace - Choix des examens d'imagerie Justification du choix des examens d'imagerie. 


\section{Introduction}

Les douleurs abdominales aiguës sont parmi les motifs les plus fréquents de consultation dans un service d'urgence hospitalière (Sturman, 1991). L'abdomen aigu est défini par l'ACR (American College of Radiology), comme une douleur nécessitant un traitement médical ou chirurgical urgent. Elles représentent environ $10 \%$ des consultations dans les centres d'urgences (Flasar et al, 2006). Une étude réalisée au Togo, a retrouvé cinq étiologies à l'origine des urgences abdominales non traumatiques, dominées par les appendicites $(36,26 \%)$ suivie des péritonites $(33,04 \%)$, des occlusions intestinales aigües $(6,43 \%)$ et des cholécystites aigues $(2,25 \%)$ (Attipou et al, 2005).

Leur diagnostic nécessite un examen soigneux et rapide. Mais, en raison de l'aspect clinique similaire de ces affections, ou du fait que l'examen clinique soit le plus souvent incomplet, des examens d'imagerie sont souvent nécessaires pour confirmer le diagnostic établi cliniquement (Cuénod et al, 1999). Depuis longtemps, dans notre pays le couple radiographie de l'abdomen sans préparation (ASP) / Echographie a servi au diagnostic de ces pathologies. Mais l'avènement de la TDM semble changer leur approche diagnostique et même étiologique. Malgré tout, certains médecins semblent toujours se contenter du couple ASP/Echographie, d'autres par contre font abusivement appel au scanner abdominal.

Ce travail a donc été initié dans le but d'évaluer la connaissance et la motivation des médecins des Centre Hospitalier Universitaire(CHU) de Lomé sur la prescription des examens d'imagerie dans la prise en charge des urgences abdominales non traumatiques les plus fréquentes au Togo, en l'occurrence les appendicites, les péritonites, les occlusions intestinales aigües et les cholécystites aigues.

\section{Materiels Et Methode}

Notre étude est transversale, descriptive et analytique qui s'est déroulée dans les centres hospitaliers universitaires (CHU) du CAMPUS et SYLVANUS OLYMPIO (SO) de Lomé (Togo) de janvier à juin 2015. Elle a consisté à la distribution d'un questionnaire aux médecins généralistes, ainsi qu'aux internes exerçant dans les deux CHU. Pour assurer la fiabilité des données, les médecins remplissaient l'auto-questionnaire en présence de l'enquêteur, sans recours à une source d'information.

La fiche d'enquête était composée de questions sur les caractéristiques de l'enquêté et des questions ouvertes et fermées à choix multiples à propos de quatre pathologies d'urgence abdominale retrouvées au Togo selon l'étude de Attipou et al (2005) à savoir : l'appendicite aigue, la péritonite aigue, les occlusions intestinales aigues et la cholécystite aigu. 
Ces questions portaient sur la fréquence du diagnostic de ces pathologies, la place de l'examen clinique et des examens d'imagerie, le choix de l'examen d'imagerie de première intention, de deuxième intention et le plus efficace pour le diagnostic de ces pathologies et les raisons ayant motivé le choix de cet examen. Des propositions ont été faites à chaque fois et la latitude était laissée au médecin d'apporter un plus lorsqu'il n'est pas satisfait de ces propositions.

L'anonymat des médecins a été assuré par la codification des questionnaires.

L'analyse des données ont été réalisés à l'aide du logiciel EPI INFO version 7 .

\section{Resultats}

Notre étude a concerné 110 médecins. L’âge moyen était de 37+/-12 ans. Le sexe masculin prédominait avec $81 \%$. Les réponses des médecins étaient variées.

Concernant 1'appendicite aigue, $72 \%$ des médecins estimaient que 1'examen clinique était suffisant pour son diagnostic. Pour 73\% des médecins l'imagerie était non nécessaire pour le diagnostic. $92 \%$ des médecins estimaient que l'échographie abdominale était l'examen d'imagerie de première intention (Tableau I).

Tableau I : Répartition des examens d'imagerie de première intention en fonction des pathologies

\begin{tabular}{|c|c|c|c|c|c|}
\hline & APPENDICITE & PERITONITE & OCCLUSION & CHOLECYSTITE & TOTAL \\
\hline ASP & $09(08 \%)$ & $108(98 \%)$ & $106(96 \%)$ & $03(03 \%)$ & $226(51,5 \%)$ \\
\hline US & $101(92 \%)$ & $02(02 \%)$ & $0(00 \%)$ & $105(95 \%)$ & $208(47 \%)$ \\
\hline TDM & $0(00 \%)$ & $0(00 \%)$ & $02(02 \%)$ & $02(02 \%)$ & $04(01 \%)$ \\
\hline IRM & $0(00 \%)$ & $0(00 \%)$ & $02(02 \%)$ & $0(00 \%)$ & $02(0,5 \%)$ \\
\hline TOTAL & $110(100 \%)$ & $110(100 \%)$ & $110(100 \%)$ & $110(100 \%)$ & $440(100 \%)$ \\
\hline \multicolumn{6}{|c|}{$\begin{array}{l}\text { L'échographie serait l'examen d'imagerie de première intention dans les appendicites }(92 \%) \\
\text { et dans les cholécystites }(95 \%) \text {, alors que l'ASP serait préconisé en première intention dans } \\
\text { les péritonites }(98 \%) \text { et dans les occlusions }(96 \%) .\end{array}$} \\
\hline \multicolumn{6}{|c|}{$\begin{array}{l}\text { Sa disponibilité était la raison qui motivait sa prescription en première } \\
\text { intention selon } 40 \% \text { des médecins, (Tableau II). } \\
\text { Tableau II : Justification de la prescription de l'examen de première intention }\end{array}$} \\
\hline
\end{tabular}

APPENDICITE PERITONITE OCCLUSION CHOLECYSTITE TOTAL 


\begin{tabular}{lccccc}
\hline Moins couteux & $29(26 \%)$ & $19(17 \%)$ & $56(51 \%)$ & $30(27 \%)$ & $134(30 \%)$ \\
Plus disponible & $44(40 \%)$ & $57(52 \%)$ & $34(31 \%)$ & $58(53 \%)$ & $193(44 \%)$ \\
Plus efficace & $37(34 \%)$ & $34(31 \%)$ & $20(18 \%)$ & $22(20 \%)$ & $113(26 \%)$ \\
TOTAL & $110(100 \%)$ & $110(100 \%)$ & $110(100 \%)$ & $110(100 \%)$ & $440(100 \%)$ \\
\hline
\end{tabular}

L'examen d'imagerie de première intention était prescrit du fait de sa disponibilité dans les appendicites (40\%), les cholécystites $58 \%$ ), et dans les péritonites $(57 \%)$ et du fait de son moindre cout dans les occlusions $(56 \%)$

Pour $87 \%$ des médecins, le scanner abdominal était l'examen le plus efficace (Tableau III).

Tableau III : Répartition des examens d'imagerie les plus efficaces en fonction des pathologies

\begin{tabular}{lccccr}
\hline & APPENDICITE & PERITONITE & OCLLUSION & CHOLECYSTITE & TOTAL \\
\hline ASP & $0(00 \%)$ & $05(05 \%)$ & $0(00 \%)$ & $0(00 \%)$ & $05(01 \%)$ \\
US & $0(00 \%)$ & $07(06 \%)$ & $0(00 \%)$ & $0(00 \%)$ & $07(02 \%)$ \\
TDM & $96(87 \%)$ & $98(89 \%)$ & $110(100 \%)$ & $106(96 \%)$ & $410(93 \%)$ \\
IRM & $14(13 \%)$ & $0(00 \%)$ & $0(00 \%)$ & $04(04 \%)$ & $18(04 \%)$ \\
TOTAL & $110(100 \%)$ & $110(100 \%)$ & $110(100 \%)$ & $110(100 \%)$ & $440(100 \%)$ \\
\hline
\end{tabular}

L'examen d'imagerie le plus efficace selon les médecins était la TDM dans toutes les quatre pathologies.

Mais $58 \%$ des médecins ne trouvaient pas nécessaire de le prescrire en première intention (Tableau IV). Il était essentiellement prescrit en cas de doute diagnostic par 78\% des médecins (Tableau V).

Tableau IV : Justification de la non prescription de l'examen le plus efficace en première intention

APPENDICITE PERITONITE OCCLUSION CHOLECYSTITE TOTAL

\begin{tabular}{lccccc}
\hline Très couteux & $33(30 \%)$ & $56(51 \%)$ & $55(50 \%)$ & $73(66 \%)$ & $217(49,5 \%)$ \\
Moins disponible & $19(17 \%)$ & $34(31 \%)$ & $33(30 \%)$ & $26(24 \%)$ & $112(25,5 \%)$ \\
Pas nécessaire & $58(53 \%)$ & $20(18 \%)$ & $22(20 \%)$ & $11(10 \%)$ & $111(25 \%)$ \\
TOTAL & $110(100 \%)$ & $110(100 \%)$ & $110(100 \%)$ & $110(100 \%)$ & $440(100 \%)$ \\
\hline
\end{tabular}

L'examen d'imagerie le plus efficace (TDM) n'était pas préconisé en première intention dans les péritonites $(51 \%)$, dans les occlusions $(50 \%)$ et dans les cholécystites $(66 \%)$ du fait de son cout élevé. Les médecins (53\%) ne trouvaient pas la TDM nécessaire dans les appendicites 
Dans les péritonites, $71 \%$ des médecins estimaient que l'examen clinique n'était pas suffisant pour le diagnostic et pour $69 \%$, l'imagerie était importante pour le diagnostic. Pour $98 \%$ des médecins l'ASP était l'examen de première intention (Tableau I). Sa grande disponibilité motivait sa prescription en première intention selon $52 \%$ des médecins (Tableau II). $89 \%$ des médecins estimaient le scanner comme l'examen le plus efficace (Tableau III). Il n'était pas préconisé en première intention du fait de son cout élevé selon $51 \%$ des médecins (Tableau IV). Il était recommandé en deuxième intention en cas de recherche d'une étiologie par 70\% des médecins (Tableau V).

Tableau V : Justification du contexte prescription de l'examen le plus efficace

\begin{tabular}{lccccc}
\multicolumn{2}{c}{ APPENDICITE } & PERITONITE & OCCLUSION & CHOLECYSTITE & TOTAL \\
\hline Confirmation du diagnostic & $86(78 \%)$ & $25(23 \%)$ & $78(71 \%)$ & $79(72 \%)$ & $268(61 \%)$ \\
Recherche étiologique & $16(15 \%)$ & $77(70 \%)$ & $22(20 \%)$ & $19(17 \%)$ & $134(30 \%)$ \\
Moyens suffisants & $08(07 \%)$ & $08(07 \%)$ & $10(09 \%)$ & $12(11 \%)$ & $38(09 \%)$ \\
TOTAL & $110(100 \%)$ & $110(100 \%)$ & $110(100 \%)$ & $10(100 \%)$ & $440(100 \%)$ \\
\hline
\end{tabular}

L'examen le plus efficace était préconisé essentiellement en cas de doute diagnostique et dans la recherche étiologique

Pour le diagnostic des occlusions intestinales, $90 \%$ des médecins estimaient que l'examen clinique n'était pas suffisant et $89 \%$ des médecins estimaient l'imagerie nécessaire. Pour 96\% des médecins, l'examen de première intention était 1'ASP (Tableau I). Sa prescription en première intention était motivée par sa plus grande disponibilité pour $51 \%$ des médecins (Tableau II). Tous les médecins estimaient que le scanner abdominal était l'examen le plus efficace (Tableau III). Il n'était pas prescrit en première intention du fait de son cout élevé pour 50\% des médecins (Tableau IV). Le scanner était préconisé dans recherche étiologique par $71 \%$ des médecins (Tableau V).

Selon 95\% des médecins, l'examen clinique ne serait pas suffisant pour le diagnostic des cholécystites aigues, et estimaient que l'imagerie est nécessaire pour le diagnostic. Pour 95\% des médecins, l'échographie abdominale était l'examen d'imagerie de première intention (Tableau I). Elle était prescrite en première intention du fait de sa disponibilité pour $50 \%$ des médecins (Tableau II). 96\% des médecins estimaient que le scanner était l'examen d'imagerie le plus efficace (Tableau III). Il n'était pas prescrit en première intention du fait de son cout élevé selon $66 \%$ des médecins (Tableau IV). Il était recommandé en deuxième intention pour la confirmation diagnostique par $72 \%$ des médecins (Tableau $\mathrm{V}$ ). 
En général, concernant les quatre pathologies des urgences abdominales étudiées, $79 \%$ des médecins de notre étude estimaient que l'examen clinique n'était pas suffisant pour poser leur diagnostic et $78 \%$ avaient recours à l'imagerie. Les examens de premières intentions étaient l'ASP (51,5\%) et l'échographie (47\%) (Tableau I). Le premier choix de ces examens d'imagerie était motivé par leur plus grande disponibilité (44\%) et par leur moindre coût (30\%) (Tableau II). Le scanner était reconnu dans 93\% des cas comme l'examen le plus efficace (Tableau III), mais son cout élevé empêcherait sa prescription en première intention dans près de la moitié des cas (Tableau IV). Il était recommandé dans 61\% des cas lorsqu'il existe un doute diagnostique (Tableau V).

\section{Discussion}

Les urgences abdominales, ou « abdomens aigus », non traumatiques s'expriment par des tableaux divers qui ont en commun l'urgence thérapeutique. Dans notre étude, $79 \%$ des médecins estimaient que 1 'examen clinique était insuffisant dans le diagnostic des urgences abdominales et $78 \%$ font systématiquement appel à l'imagerie. Les séries cliniques font état de 25 à 50\% d'erreurs de diagnostic quand on ne fait pas appel à l'imagerie (Mindelzun et al, 1997). En effet, les progrès de l'imagerie aboutissent à des informations de plus en plus précises sur l'ensemble des pathologies très diverses susceptibles de causer une douleur abdominale. A côté des classiques clichés de l'abdomen sans préparation, l'imagerie en coupes a pris une part de plus en plus importante par son impact sur le diagnostic et le traitement.

Concernant l'appendicite aigue, les signes cliniques ne sont retrouvés que chez environ 50\% des patients (Rettenbacher et al, 2002). Pourtant dans notre étude $72 \%$ des médecins estimaient que l'examen clinique peut être suffisant pour son diagnostic et $73 \%$ trouvaient l'imagerie non nécessaire. Il est vrai que le diagnostic d'appendicite a été longtemps considéré comme exclusivement clinique. Cette attitude conduit a' un retard diagnostique, responsable de complications (Taourel et al, 2002). Il est maintenant recommandé de confirmer le diagnostic d'appendicite aiguë par l'imagerie avant toute chirurgie. L'échographie, le scanner et l'IRM sont les trois techniques capables de faire le diagnostic d'appendicite aigue. Dans notre étude 92\% des médecins estimaient que l'échographie est l'examen de première intention. Aucun médecin n'avait recommandé le scanner en première intention. En effet, l'échographie est à privilégier en première intention. Sa sensibilité est évaluée entre 78 et $83 \%$ et sa spécificité entre 83 et 93\% (Doria et al, 2006 ; van Randen et al, 2008). Le cliché d'ASP n'a en revanche aucune place dans le diagnostic de l'appendicite, à cause de sa faible sensibilité et spécificité. Dans notre étude, $87 \%$ des médecins estiment que le scanner est l'examen le plus efficace. Le scanner est en effet l'examen de 
prédilection pour le diagnostic d'appendicite aiguë avec une sensibilité de 91 à $94 \%$ et une spécificité de 90 à $94 \%$ (Doria et al, 2006 ; van Randen et al, 2008). Il est supérieur à l'échographie en terme de sensibilité (Doria et al, 2006 ; van Randen et al, 2008). Mais, le scanner ne serait recommandé en première intention à cause de son irradiation Cobben et al, 2009). Il est particulièrement recommandé dans les appendicites compliquées ou ectopiques, ou chez lez les patients obèses (Balthazar et al, 1994). Mais, selon une autre étude Américaine (Rao et al, 1998), la réalisation systématique d'un scanner en première intention devant tout syndrome appendiculaire permettrait d'importantes économies de santé en évitant une appendicectomie dans $13 \%$ des cas et une surveillance inutile dans $39 \%$ des cas.

Cliniquement, le diagnostic des péritonites ne serait pas facile comme le témoigne $71 \%$ des médecins de notre étude. L'imagerie va alors jouer un rôle important dans le diagnostic de cette pathologie. $69 \%$ des médecins de notre étude ont systématiquement recourt à l'imagerie devant une péritonite aigue. L'ASP, complété par le cliché de thorax debout et de face, permet le plus souvent la détection de pneumopéritoine en permettant de visualiser un pneumopéritoine. Ceci en fait l'examen de première intention (Taourel et al, 2001). Dans notre étude $98 \%$ des médecins prescrivent l'ASP en première intention devant une suspicion de péritonite aiguë, compte tenu de sa disponibilité et son moindre coût. La sensibilité de l'ASP serait de $56 \%$ sur la base des clichés de face en position couchée, de $76 \%$ en position debout et de 90\% si l'on utilise en plus le cliché de décubitus latéral gauche (Krestin et al, 1993). Mais, parfois, de tous petits pneumopéritoines peuvent ne pas être identifiés sur les radiographies conventionnelles, mais retrouvés uniquement par le scanner (Taourel et al, 2001). Dans notre étude 98\% des médecins estimaient que le scanner est plus efficace et le préconise en 2ème intention en cas de doute diagnostic. Dans une série rétrospective de 10 cas d'ulcère gastroduodénal perforé ayant bénéficié d'un ASP et d'une TDM, l'ASP n'a trouvé le pneumopéritoine que dans 2 cas alors qu'il était toujours visualisé en TDM (Ongolo-Zogo et al, 1999). Lorsque la perforation est cloisonnée, des images aériques extra-digestives peuvent être difficiles à affirmer sur l'ASP. La TDM montre le caractère extra-digestif de l'air et permet d'évoquer le siège de la perforation devant le siège des bulles d'air extradigestif ou devant des anomalies pariétales digestives. L'échographie, va servir à mettre en évidence un épanchement liquidien intrapéritonéal, mais elle est peu performante pour détecter un pneumopéritoine (Lee et al, 1990). Seulement $2 \%$ des médecins de notre étude la demandent en première intention.

Dans les occlusions intestinales (OI), les signes cliniques seraient insuffisants pour le diagnostic (Taourel et al, 2001), comme le confirment 90\% des médecins de notre étude. Selon $96 \%$ des médecins de notre étude, l'ASP serait l'examen de première intention devant une OI aiguë. L'ASP peut être 
en effet, réalisée en première intention dans les OI, car, elle est facilement accessible et peut rapidement orienter le diagnostic (Taourel et al, 2001). Mais son rendement serait faible (Thompson et al, 2007). De même, l'ASP est souvent insuffisante pour le diagnostic des complications et de l'étiologie des OI. Selon le guide de bon usage des examens d'imagerie médicale de la société française de radiologie (SFR), l'ASP est non indiqué dans le diagnostic des OI et le scanner est l'examen de première intention. Tous les médecins de notre étude avaient reconnu la supériorité du scanner, mais le préconisent en 2ème intention compte tenu de son coût élevé et son indisponibilité en urgence. Le scanner est en effet, plus performant avec une fiabilité supérieure à $90 \%$ pour le diagnostic positif et de siège. Il a une sensibilité de 90 à $94 \%$ et une spécificité de $96 \%$ pour le diagnostic d'occlusion mécanique du grêle (Cuénod et al, 1999). Il permet également d'identifier la cause de l'occlusion dans 73\% des cas et de diagnostiquer les signes de gravité (Megibow et al, 1991). L'échographie reste encore assez anecdotique dans le diagnostic d'occlusion (Taourel et al, 2001). Seulement 2\% des médecins de notre étude ont préconisé sa réalisation.

Les signes cliniques, en cas de cholécystite, sont le plus souvent incomplets (Lameris et al, 2008). Ainsi, 95\% des médecins de notre étude estimaient les signes cliniques non suffisants pour le diagnostic. L'imagerie va donc occuper une place importante. $95 \%$ des médecins de notre étude recommandent la prescription de l'échographie en première intention. En effet, selon l'ACR, l'échographie est la modalité de choix et la plus appropriée pour le diagnostic de cholécystite aiguë. Elle reste ainsi la technique d'imagerie de première intention et de référence dans cette situation clinique et doit être réalisée en urgence selon le guide de bon usage des examens d'imagerie de la SFR. Sa sensibilité serait de $88 \%$ et sa spécificité de $80 \%$ (Shea et al, 1994). Pour 96\% des médecins de notre étude, le scanner est l'examen le plus efficace dans le diagnostic des cholécystites. Ceci est en contradiction avec les données de la littérature. En effet, selon le guide de bon usage des examens d'imagerie médicale de la SFR, le scanner a un rôle restreint dans le diagnostic des cholécystites. Mais selon une étude, le scanner serait performant, avec une sensibilité de $92 \%$ et une spécificité de $99 \%$ (Bennett et al, 2002). Mais la plupart des études s'accordent sur le fait que l'échographie est la modalité d'imagerie à prescrire en cas de suspicion de cholécystite (Bree et al, 2000). Le scanner serait recommandé devant des complications et lorsque l'obésité devient un facteur limitant de l'échographique de qualité.

En général, l'ASP et l'Echographie sont les deux examens les plus prescrits en première intention dans la pratique quotidienne des médecins de notre étude en raison de leur disponibilité et de leur coût moindre. Il est donc évident que les examens prescrits dans notre étude ne tiennent pas compte de 
l'efficacité, mais plutôt de la disponibilité et surtout du coût de l'examen. En effet, l'ASP est jugé obsolète et non indiqué dans les urgences abdominales selon le guide de bon usage des examens d'imagerie de la SFR. Ce guide ne recommande l'échographie en première intention que dans les cholécystites et dans les appendicites. Selon ce guide, le scanner est l'examen de première intention dans les urgences abdominales en dehors de la cholécystite et dans certains cas d'appendicite. Dans un travail récent (Sievert et al, 1997), le scanner avait une sensibilité diagnostique de $90 \%$ dans les abdomens aigus. Les données de la TDM entraînaient une modification de l'attitude thérapeutique décidée initialement chez $27 \%$ des malades (Sievert et al, 1997). Mais, selon près de la moitié des médecins de notre étude, la cherté du scanner serait un frein à sa prescription en première intention et pour près de $26 \%$, son indisponibilité limiterait sa prescription en urgence. Donc la prescription du scanner en première intention semble être difficile à mettre en œuvre en Afrique sub-saharienne. Pourtant le coût élevé du scanner ne doit en principe pas être un frein à la prescription du scanner en première intention, car selon une étude (Rosen et al, 2000), la prescription du scanner en première intention permettrait de faire des économies, en permettant d'éviter des diagnostics tardifs et en permettant une prise en charge rapide avant la survenue des complications. Ceci permet un raccourcissement de la durée d'hospitalisation (Rosen et al, 2000). Donc le scanner peut être envisagé comme examen de première intention dans les villes et les hôpitaux de nos pays qui en disposent. Dans notre étude, $61 \%$ des médecins prescrivent le scanner en cas de doute persistant sur le diagnostic après le premier examen. Mais le recourt au scanner en deuxième intention, devant le manque d'efficacité du premier examen prescrit est le plus souvent source de dépenses supplémentaires et de retard diagnostic.

\section{Conclusion}

Les urgences abdominales posent encore un problème diagnostic dans les pays en voies de développement. Les examens d'imagerie qui permettent de faire le diagnostic ne sont pas prescrits en fonction de leur efficacité, mais en fonction de leur disponibilité et de leur coût. Ce qui empêche le plus souvent la prescription du scanner qui est l'examen d'imagerie le plus efficace, mais le plus cher et le moins disponible en urgence dans les pays en voies de développement. Aussi, l'absence de l'assurance maladie dans ces pays représente un frein à la prescription de cet examen. Ainsi, l'ASP qui semble être obsolète est l'examen le plus prescrit, suivi de l'échographie qui n'est efficace que dans certains cas d'appendicite et dans les cholécystites. 


\section{Déclaration d'intérêts}

Les auteurs déclarent ne pas avoir de conflits d'intérêts en relation avec cet article.

\section{References:}

1. Sturman M. Medical imaging in acute abdominal pain. Compr Ther 1991; 17:15-21.

2. Flasar MH, Goldberg E. Acute abdominal pain. Med Clin N Am 2006;90:481-503.

3. Attipou K, Kanassoua K, Dosseh D. Urgences chirurgicales abdominales non traumatiques de l'adulte au Chu Todoin de Lome (Bilan de 5 annees). J Rech Scient UL 2005; 7:

4. Cuénod CA, Wind P, Siauve N, Clément O, Bénichou J, Frija G. Diagnostic des urgences abdominales aiguës d'origine digestive chez l'adulte. Gastroentérol clin biol 1999; 23: 1170

5. Mindelzun RE, Jeffrey RB. Unenhanced helical CT for, evaluating acute abdominal pain: « A little morecost, a lot more information ». Radiology 1997; 205: 43-47.

6. Rettenbacher T, Hollerweger A, Gritzmann N, et al. Appendicitis: should diagnostic imaging be performed if the clinical presentation is highly suggestive of the disease? Gastroenterology 2002;123:992-8.

7. Taourel P, Kessler N, Blayac PM, Lesnik A, Gallix B, Bruel JM. Imagerie de l'appendicite : échographie, scanner ou rien du tout ? J Radiol 2002;83:1952-60.

8. Doria AS, Moineddin R, Kellenberger CJ, et al. US or CT for Diagnosis of Appendicitis in Children and Adults? A Meta-Analysis. Radiology 2006;241:83-94.

9. van Randen A, Bipat S, Zwinderman AH, Ubbink DT, Stoker J, Boermeester MA. Acute appendicitis: meta-analysis of diagnostic performance of CT and graded compression US related to prevalence of disease. Radiology 2008;249:97-106.

10. Cobben L, Groot I, Kingma L, Coerkamp E, Puylaert J, Blickman J. A simple MRI protocol in patients with clinically suspected appendicitis: results in 138 patients and effect on outcome of appendectomy. Eur Radiol 2009; 19:1175-83.

11. Balthazar E, Birnbaum B, Yee J, Megibow A, Roshkow J, Gray C. Acute appendicitis : CT and US correlation in 100 patients. Radiology 1994; 190:31-5.

12. Rao P, Rhea J, Novelline R, Mostafavi A, McCabe C. Effect of computed tomography of the appendix on treatment of patients and use of hospital resources. N Engl J Med 1998; 338:141-6. 
13. Taourel P, Bruel JM Apport de l'imagerie dans les urgences du tube digestif. Gastroentérologie clinique \& biologique2001; 25: 178-182

14. Krestin GP, Choyke PL. Acute abdomen: diagnostic imaging in the clinical context. Georg Thieme Verlag, Thieme Medical Publishers, Inc. Stuttgart, New York. 1996

15. Ongolo-Zogo P, Borson O, Garcia P, Grüner L, Valette PJ. Acute gastro-duodenal peptic ulcer perforation: contrast enhanced and thinsection spiral: CT findings in 10 patients. Abdom Imaging 1999; 24:329-32.

16. Lee DH, Lim JH, Ko YT, Yoon Y. Sonographic detection of pneumoperitoneum in patients with acute abdomen. AJR $1990 ; 154$ : $107-9$

17. Thompson WM, Kilani RK, Smith BB, Thomas J, Jaffe TA, DeLong DM, Paulson EK. Accuracy of abdominal radiography in acute smallbowel obstruction: does reviewer experience matter? AJR Am J Roentgenol 2007 ; 188 : 233-8

18. Megibow A, Balthazar E, Cho K, Medwid S, Birnbaum B, Noz M. Bowel obstruction : evaluation with CT. Radiology 1991;180:313-8.

19. Lameris W, van Randen A, Ten Hove W, Bossuyt PM, Boermeester MA, Stoker J. The clinical diagnosis of acute cholecystitis is unreliable [abstr]. In: Radiological Society of North America Scientific Assembly and Annual Meeting Program. Oak Brook, Ill: Radiological Society of North America, 2008; 110.

20. Shea JA, Berlin JA, Escarce JJ, et al. Revised estimates of diagnostic test sensitivity and specificity in suspected biliary tract disease. Arch Intern Med 1994;154:2573-2581.

21. Bennett GL, Rusinek H, Lisi V, et al. CT findings in acute gangrenous cholecystitis. AJR Am J Roentgenol 2002;178:275-281.

22. Bree RL, Ralls PW, Balfe DM, et al. Evaluation of patients with acute right upper quadrant pain: American College of Radiology - ACR appropriateness criteria. Radiology 2000; 215(suppl):153-157.

23. Sievert B, Raptopoulos V, Mueller M, Rosen M, Steer M. Impact of CT on diagnosis and management of acute abdomen in patients initially treated without surgery. Am J Roentgenol 1997; 168:173-8.

24. Rosen M. Impact of Abdominal CT on the Management of Patients Presenting to the Emergency Department with Acute Abdominal Pain. AJR 2000; 174: 1391-6. 